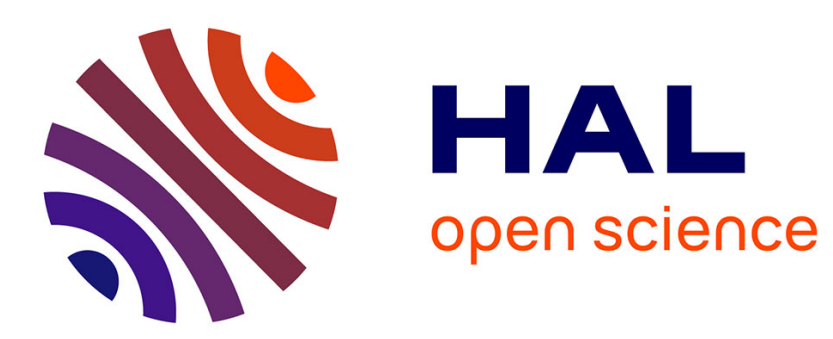

\title{
Critical fouling conditions induced by colloidal surface interaction: from causes to consequences
}

Patrice Bacchin, Pierre Aimar

\section{To cite this version:}

Patrice Bacchin, Pierre Aimar. Critical fouling conditions induced by colloidal surface interaction: from causes to consequences. Desalination, 2005, 175 (1), pp.21-27. 10.1016/j.desal.2004.09.020 . hal-00201064

\section{HAL Id: hal-00201064 \\ https://hal.science/hal-00201064}

Submitted on 22 Dec 2007

HAL is a multi-disciplinary open access archive for the deposit and dissemination of scientific research documents, whether they are published or not. The documents may come from teaching and research institutions in France or abroad, or from public or private research centers.
L'archive ouverte pluridisciplinaire HAL, est destinée au dépôt et à la diffusion de documents scientifiques de niveau recherche, publiés ou non, émanant des établissements d'enseignement et de recherche français ou étrangers, des laboratoires publics ou privés. 


\title{
Critical fouling conditions induced by colloidal surface interaction: from causes to consequences
}

\author{
P. Bacchin ${ }^{*}$, P. Aimar \\ Laboratoire de Génie Chimique, Université Paul Sabatier, 118 route de Narbonne,
}

31062 Toulouse Cedex, France

*Corresponding author bacchin@chimie ups-tlse.fr, Fax number (3.3) 561556139

\begin{abstract}
Critical fouling conditions (CFC) are defined as the process operating conditions leading to the formation of multilayer irreversible fouling at the membrane surface. This irreversible fouling is considered as the result of a phase transition in the accumulated matter from a dispersed phase (concentration polarisation) to a condensed phase (deposit or gel formation) : the spinodal decomposition. Properties of concentrated colloid dispersions and their related phase transitions are integrated in a classical filtration mass balance via the colloidal osmotic pressure, $\Pi$. This then allows us to define critical fouling conditions for both cross flow and dead end filtration. These critical fouling conditions (CFC) are expressed in term of critical pairs of operating conditions: the set permeate flux / boundary layer thickness (directly linked to cross flow velocity) in cross flow and the critical set permeate flux / filtered volume in dead end.
\end{abstract}

Keywords : colloid, fouling, osmotic pressure, phase diagram, ultrafiltration

\section{Introduction}

Mass accumulation at a membrane surface is the natural consequence of the separation. Such an accumulation can be reduced by a tangential flow (i.e. by using energy) but never totally avoided. This accumulation leads to a reduction of permeate flux (a decrease in productivity) but also requires to operate periodical membrane cleaning in order to maintain an overall good productivity. But the efficiency of the cleaning step depends on the way accumulation occurs.

First, accumulation takes place in the form of concentration polarisation i.e. mass accumulated in a dispersed phase on the membrane surface. Concentration polarisation layer is totally reversible with relaxation of the separation force.

Accumulation can also induce a fouling characterised by its irreversibility (if the separation force is decreased). Fouling can be differentiated according to its location:

- internal fouling i.e. fouling inside the porous medium (pore plugging, internal adsorption)

- interfacial (monolayer) fouling resulting of interfacial interaction between the material and the solute (adsorption, pore blinding).

- superficial (multilayer) fouling i.e. a growth in fouling layer from the membrane surface toward the bulk. In contrast to concentration polarisation, fouling is made of matter in a condensed state. This form of fouling corresponds to gel or deposit layer which can be considered as irreversible when the separation force is relaxed (or sometimes weakly reversible after a long time at rest-relaxation phenomena - ). 
Critical fouling conditions (CFC) are defined as process conditions beyond which multilayer of fouling growth at the membrane surface. The growth of this cohesive matter on the membrane surface needs a significant amount of energy to be removed. Beneath CFC, accumulation is totally reversible (concentration polarisation) or fouling limited to a monolayer (interfacial fouling as adsorption) or internal fouling (that can be reduced by a correct choice of membrane cut-off). CFC are then important conditions when running a process in an optimum way. Furthermore, CFC can represent a limit beyond which significant energy is systematically required to restore the initial state whereas below these conditions only diffusion process (natural and free of charge) can allow to recover the flux. CFC could then represent an interesting working point in regard to process sustainability.

In this paper, superficial multilayer fouling is investigated theoretically first by analysing its physical causes, secondly by proposing a way to describe these cause in order to draw consequences on the process which are defined in a third point both for cross flow and in dead end filtration.

\section{Cause: a phase transition called spinodal decomposition}

When ultrafiltering a dispersion, fouling is often the consequence of the concentration of colloids (macromolecules or sub micronic particle). Now, colloid dispersions exhibit a specific behaviour because of surface interactions. These surface interaction are multiple in nature (electrostatic repulsion, Van der Waals attraction or hydrophobic-philic interaction) and have different interaction lengths ; thus leading to a complex system when colloidal dispersions are concentrated. When investigating properties of concentrated colloids it can be useful to consider the phase diagram [1] as shown in Fig. 1. Five different phases appear relative to the concentration ( $\mathrm{x}$ axis) and the degree of destabilisation ( $\mathrm{y}$ axis) :

- "gas" phase : diluted dispersion of stable particles having a free and random motion

- "liquid" phase : network of stable colloids interacting by repulsion and then moving from and towards equilibrium position (if particles are monodisperse the network is ordered : colloidal liquid crystal)

- aggregate phase: diluted suspension of aggregate

- gel phase : network of colloids interacting by attraction characterised by an elastic behaviour

- solid phase : solid structure where colloids are in contact (with different compacity according to the fractal dimension of the structure)

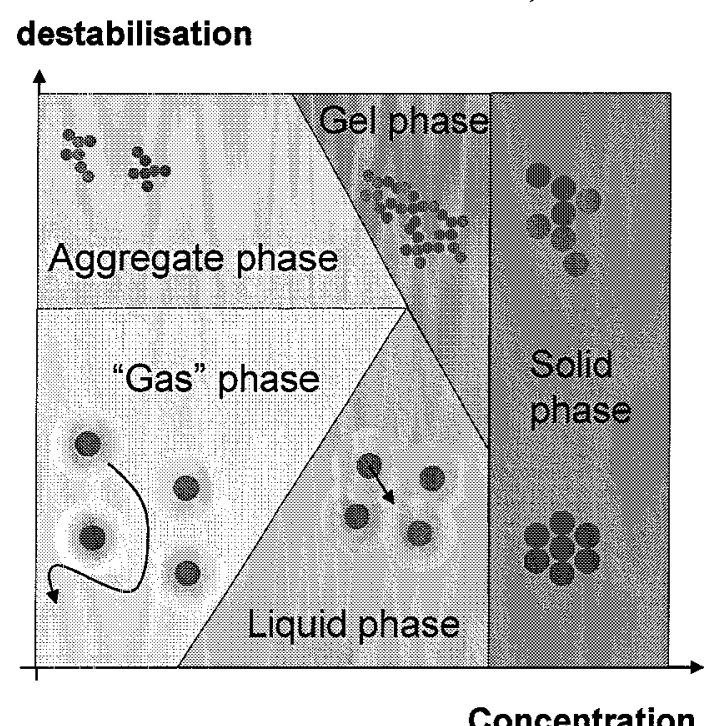

Figure 1: Schematised phase diagram of a colloidal dispersion 
The boundaries between these areas correspond to phase transitions. The transition separating the gas phase from the aggregate phase is relative to an aggregation phenomenon that occurs when dispersion is destabilised (by adding a salt or a coagulant). When concentrating these phases, the creation of the liquid or the gel phase is due to the percolation of surface interactions leading to a network of interacting colloids (by repulsion for the liquid phase and by attraction for the gel). If concentrated again, these phases undergo a spinodal decomposition i.e. an irreversible transition leading to a solid structure where colloids are in contact and interact with strong Van der Waals attractions (perfect sink of potential energy).

One can appreciate here the fundamental importance of such phase transitions on the way irreversible fouling forms on a membrane. But a model is needed to account for this behaviour in the description of transport phenomena occurring in the filtration operation.

\section{From causes to consequences: $\Pi$-based modelling}

\subsection{Colloidal osmotic pressure}

It is possible to account for properties of concentrated colloidal dispersions via osmotic pressure, $\Pi$. Osmotic pressure is already well known in membrane processes as the main limiting phenomena when dealing with salt retention in reverse osmosis processes. But colloidal osmotic pressure has been shown as an interesting macroscopic tool to relate colloidal properties both in theoretical [2] and in experimental [3,4] points of view. Furthermore, osmotic pressure variations can easily give information about the spinodal decomposition (see previous section) that appears to be theoretically described as a maximum in the osmotic pressure versus volume fraction curve [5]. A classical variation in osmotic pressure is presented in Fig. 2 as a function of the volume fraction from computation accounting for contribution of entropy (or Brownian diffusion), repulsive electrostatic repulsion and attractive Van der Waals interaction $[2,6]$.

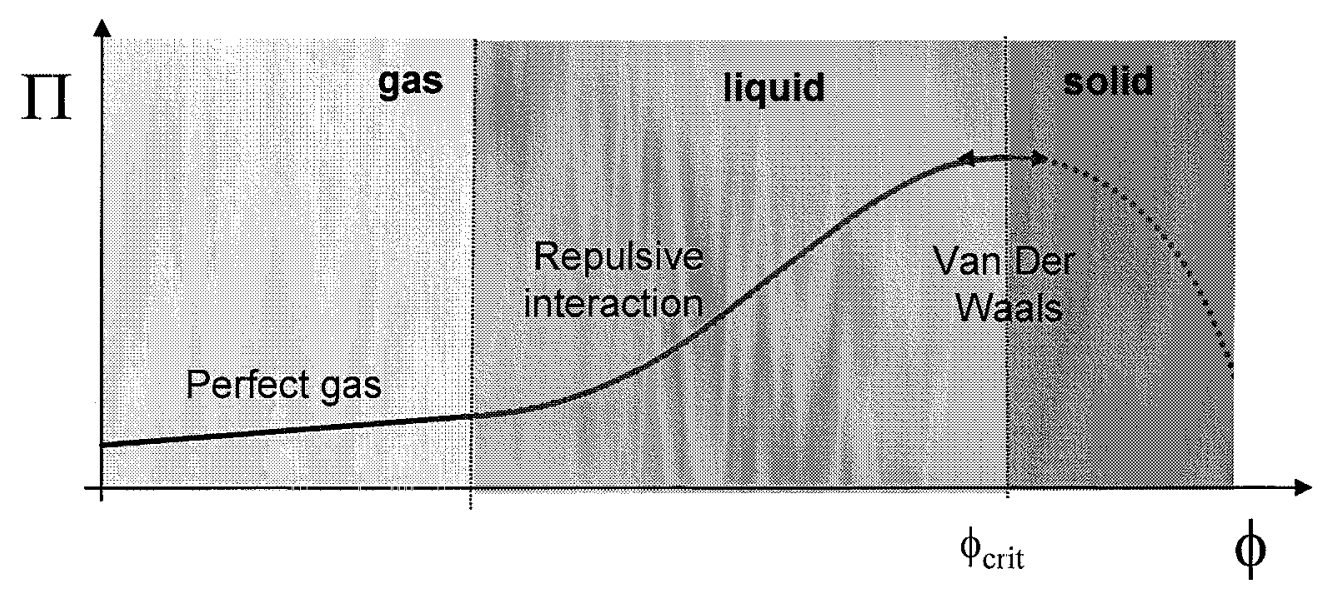

Figure $2 \cdot$ Classical variation of osmotic pressure with volume fraction for stable colloids

The first leg is a linear variation of osmotic pressure with volume fraction (Van't Hoff relationship) and corresponds to the gas phase limited by entropic contribution. When colloids are so concentrated that repulsion between particles occurs, the osmotic pressure increases more severely (gas/liquid reversible transition). The osmotic pressure curve presents then an inflexion and, later, a maximum because of the increasing part of Van der Waals attraction. A maximum (spinodal decomposition) appears when attraction exactly counterbalances repulsions thus leading to an instability: formation of aggregates. The intensity of the 
maximum of osmotic pressure has been experimentally found around 0.2 bar for $120 \mathrm{~nm}$ particles size [4]. Osmotic pressure can then be significant when compared to the classical pressure used in ultrafiltration. However, the effect of osmotic pressure does not end at the classical effect of a reduction of applied pressure accounted by using $\Delta \mathrm{P}-\Delta \Pi$ in the filtration law ; indeed, colloidal osmotic pressure is also directly linked to the diffusion phenomena and then acts on the way colloids concentrate at the membrane surface.

\subsection{Gradient (or collective) diffusion}

The gradient diffusion (defined as the collective diffusion of colloids in a concentration gradient in contrast with self diffusion) is directly linked to the variation in osmotic pressure, $\Pi$, with volume fraction, $\phi$, by the Stokes Einstein relationship [7] :

$$
D(\phi)=D_{b} K(\phi) \frac{d \Pi^{*}}{d \phi} \text { with } \Pi^{*}=\Pi \frac{V_{p}}{k_{B} T}
$$

where $K(\phi)$ is the hindered settling coefficient, $D_{b}$ is the bulk diffusion coefficient (for infinite dilution) and $V_{p}$ the volume of the colloid. By using this relationship, one can deduce the gradient diffusion coefficient (Fig. 3) corresponding to osmotic pressure variations as plotted in Fig. 2.

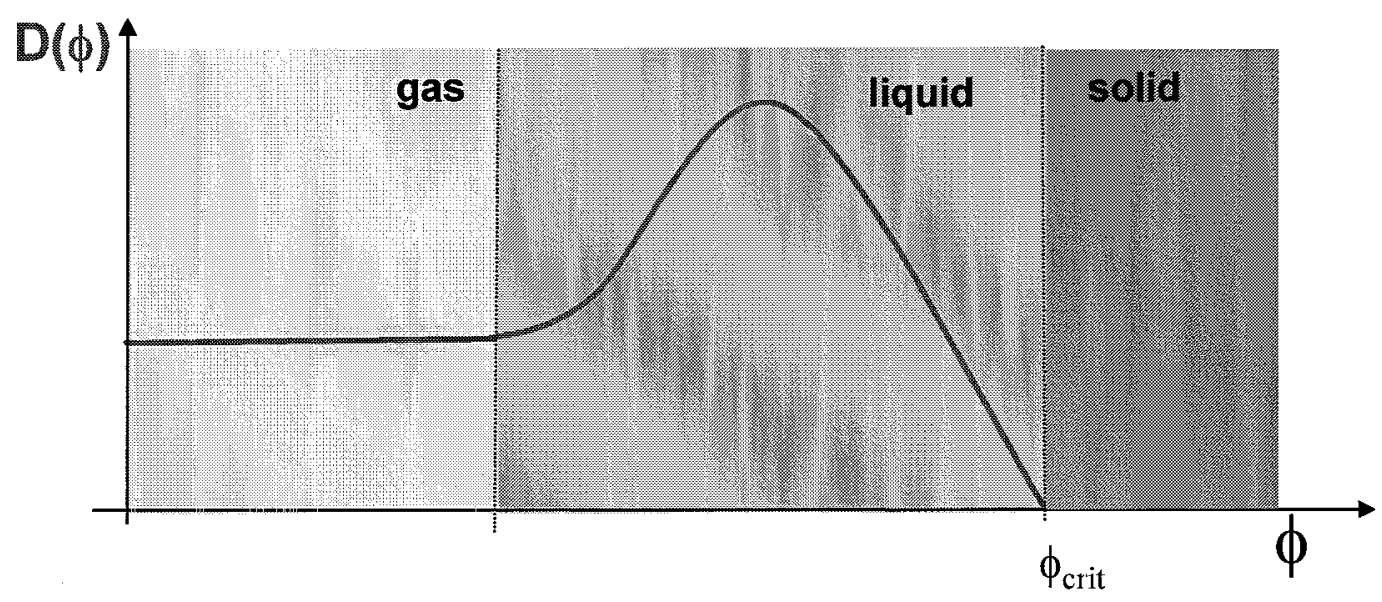

Figure 3 Evolution of collective diffusion coefficient with volume fraction relative to osmotic pressure variation shown in fig. 2.

Diffusion coefficient is seen to be constant for diluted dispersion (gas phase). Then, the gradient diffusion increases rapidly because of the concentration of particles being in repulsive interaction (repulsion acts as compressed springs inducing important collective diffusion particles). Oppositely, for higher volume fraction when attractions become important, diffusion coefficient is reduced. The diffusion is reduced to zero when the spinodal decomposition occurs (this nil value is synonym of irreversibility: the mass can not return naturally in the bulk by diffusion).

It has to be noted that osmotic pressure and diffusion coefficient variations vary depending on surface properties (zeta potential), dispersant properties (ionic strength) and particles size. For smaller size than the one used in preceding plot, the concentration lead to a progressive phase transition from gas to gel (and not liquid as noted for larger particles schematised by Figs. 2 and 3) before the transition to the solid phase. This difference can be seen as the natural causes for the formation of gel or deposit layer respectively for small macromolecules and large colloidal particles [6]. 


\section{3. $\Pi$-based filtration mass balance}

The osmotic pressure and the relative gradient diffusion coefficient can be used in classical mass balance to account for the properties of concentrated colloidal dispersion. During filtration with a fully retentive membrane the mass balance can be written as:

$$
J \phi-D(\phi) \frac{d \phi}{d x}=0
$$

These mass balance combined with the Stokes Einstein law (in Eq. (1)) leads to:

$$
\phi d x=\frac{D_{b}}{J} K(\phi) d \Pi^{*}
$$

where the accumulation (left term) is related to variations of the osmotic pressure, $\Pi(\phi)$, and the settling coefficient, $K(\phi)$, with volume fraction (right term) which depict the resistance of a dispersion to a local over concentration. These properties of the colloidal dispersion appear then very pertinent for the description of filtration processes. Consequences of Eq. (3) in transport phenomena occurring during filtration are detailed in next section.

\section{Consequences: Critical fouling conditions}

\subsection{Cross flow mode}

During filtration in cross flow mode, one considers that accumulation occurs in a fixed mass boundary layer ; the thickness of this mass boundary layer being relative to the cross flow velocity sweeping membrane wall. Eq. (3) can be integrated over this a priori known thickness, $\delta$, leading to the following relationship:

$$
\frac{J \delta}{D_{b}}=\int_{\Pi_{b}^{*}}^{\Pi_{m}^{*}} \frac{K(\phi)}{\phi} d \Pi^{*}
$$

Physically, this equation links operating conditions (left term) - i.e. permeate flux and cross flow velocity (through $\delta$ ) - to dispersion properties (right term) - i.e. osmotic pressure and settling coefficient. The left term can be seen as a Péclet number relating the importance of the friction acting on a particles along the boundary layer over the diffusion. One can define a critical Pe number leading the membrane osmotic pressure, $\Pi_{m}$, to reach the critical osmotic pressure, $\Pi_{\text {crit, }}$ as follows:

$$
P e_{c r i t}=\frac{(J \delta)_{c r i t}}{D_{b}}=\int_{\Pi_{b}^{*}}^{\Pi_{c r i t}^{*}} \frac{K(\phi)}{\phi} d \Pi^{*}
$$

The right term is then relative to the stability of the suspension when submitted to a cross flow filtration. A consequence of the critical Péclet number is that there exists a critical permeate flux for a given cross flow velocity - and then known $\delta$ - (Fig. 4b) or a critical cross flow velocity for a given permeate flux. The concept of critical Péclet number, first described in [8], can explain the way irreversible fouling layers form along a membrane channel by considering the growth of boundary layer [6]. It is then possible to have a steady flux without any superficial fouling in cross flow filtration: sub-critical operation.

\subsection{Dead end mode}

In unstirred dead end filtration, the built-up mass can be easily deduced from experiments by a mass balance between the initial solution and the collected permeate. This accumulated mass can be directly linked (via the colloid density and membrane area) to an accumulated volume of mass $V_{a}$ per square meter of membrane area $\left(\mathrm{m}^{3} / \mathrm{m}^{2}\right)$ being linked to the easily measured filtered volume per membrane area, $\mathrm{V}$, as follows: 


$$
V_{a}=R \cdot V \cdot \phi_{b}
$$

where $\mathrm{R}$ is the membrane retention coefficient and $\phi_{b}$ is the colloidal volume fraction in the bulk. Integration of Eq. (3) is then achieved over the accumulated volume of matter as follows

$$
\frac{J V_{\alpha}}{D_{b}}=\int_{\Pi_{b}^{*}}^{\Pi_{m}^{*}} K(\phi) d \Pi^{*}
$$

where the first term is relative to the set of operating conditions (permeate flux and accumulated volume of matter) and the second one is linked to the dispersion properties through the osmotic pressure and the settling coefficient. The left hand term can again be assimilated to a Péclet number relative to the ratio of friction across the whole layer accumulated particles over the diffusion. A critical Pe number can be defined when operating conditions make the osmotic pressure reach its critical value:

$$
P e_{\text {crit }}=\frac{\left(J \cdot V_{a}\right)_{c r i t}}{D_{b}}=\int_{\Pi_{b}^{*}}^{\Pi_{c r i t}^{*}} K(\phi) d \Pi^{*}
$$

Consequences of this critical Péclet number is that there should exist a critical filtered volume for a given permeate flux (Fig. 4a) and a critical permeate flux for a fixed filtered volume. Such a statement has been theoretically demonstrated when modelling filtration by a force balance approach [9]. Consequences of a critical filtered volume on dead end filtration have been investigated [10] when operating under constant pressure. Experimental evidences have been shown on latex suspension [9, 11], clays suspensions [11] and natural organic matter [12]. It is then possible to run successive dead end filtration and drain cycles with no significant formation of multilayer fouling on the membrane if processing a filtered volume under its critical value.

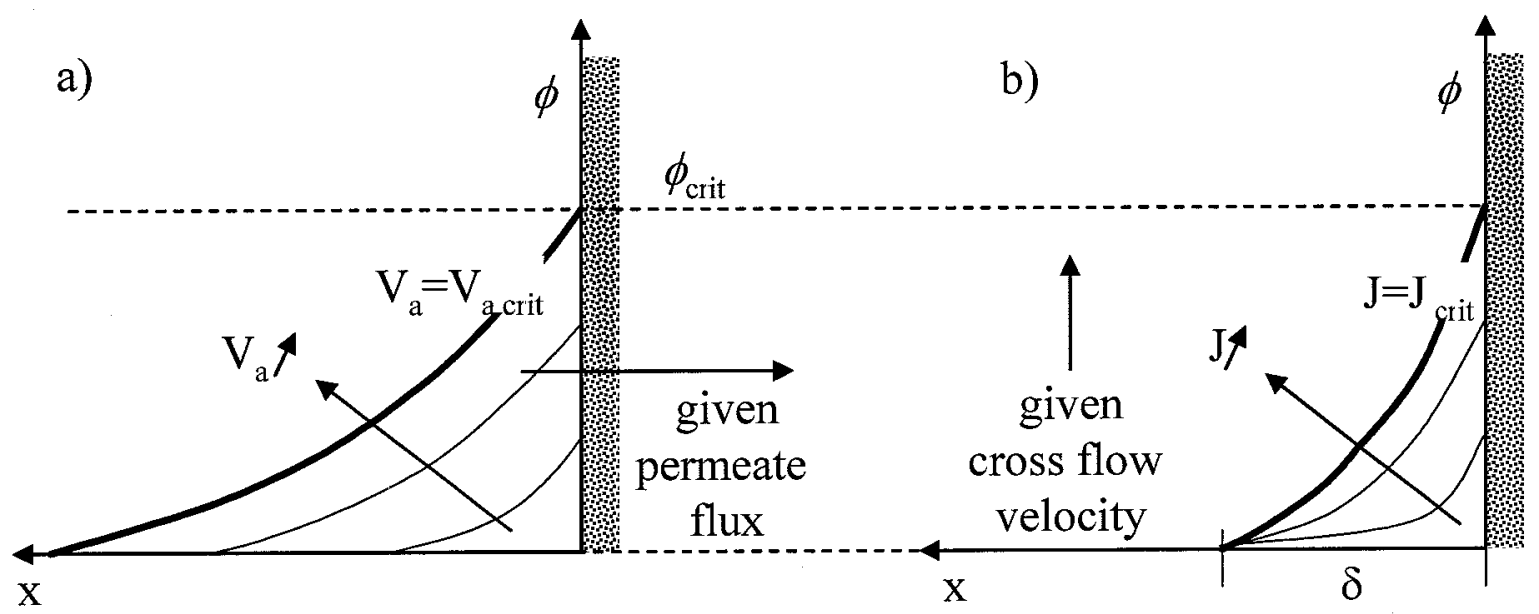

Figure 4 Schematic representation of volume fraction, $\phi$, variation with the distance to the membrane, $x$. Critical fouling conditions appears for a critical accumulated volume in dead end filtration a) or for a critical flux in cross flow filtration b)

\section{Conclusions}

The critical flux concept and its related critical fouling conditions, defined as operating conditions leading to the formation of multilayer irreversible fouling, are shown linked to properties of concentrated colloidal dispersions. An irreversible transition from a dispersed phase to a condensed phase (called spinodal decomposition by physicist) is the cause for the formation of multilayer irreversible colloidal fouling. It appears possible to account for the 
properties of concentrated colloid dispersion using a classical filtration mass balance through the colloidal osmotic pressure, П. П-based modelling allows us then to quantify critical fouling conditions both for cross flow and dead filtration. Such critical fouling conditions (CFC) are expressed in terms of a set of critical operating conditions: the set permeate flux / boundary layer thickness (directly linked to cross flow velocity) in cross flow and the set permeate flux / filtered volume in dead end.

\section{References}

[1] V J. Anderson, H.N.W. Lekkerkerker, Insights into phase transition kinetics from colloid science, Nature, 416, 6883: (2002) 811-815.

[2] A.S. Jönsson, B. Jönsson, Ultrafiltration of colloidal dispersions - A theoretical model of the concentration polarization phenomena, J. Colloid Interface Sci., 180 (1996) 504-518

[3] C. Bonnet-Gonnet, L.Belloni, B Cabane, Osmotic pressure of latex dispersions, Langmuir, 10,11 (1994), 4012-21.

[4] B. Espinasse, Approche théorique et expérimentale de la filtration tangentielle de colloïdes : flux critique et colmatage, Thèse de l'université Paul Sabatier, Toulouse, France, 2003. (Available in pdf format by request to authors).

[5] W.B. Russel, D.A. Saville, W.R. Schowalter, Colloidal Dispersions, Cambridge University Press, Cambridge (1991).

[6] P. Bacchin, D. Si-Hassen, V. Starov, M.J. Clifton, P. Aimar, A unifying model for concentration polarization gel-layer formation and particle deposition in cross-flow membrane filtration of colloidal suspensions, Chem. Eng. Sci., 75 (2002) 77-91

[7] A. Einstein, Investigation on the theory of the Brownian movement, ed. R. Furth, New York : Dover Publications, (1956)

[8] P. Bacchin, P. Aimar, V. Sanchez. Model for colloidal fouling of membranes, AIChE J, 41, (1995) 368376.

[9] P. Harmant, Contrôle de la structure de dépôts de particules colloïdales en filtration frontale et tangentielle, Thèse de l'Université Paul Sabatier, 2505 (1996)

[10] P. Bacchin, M. Meireles, P. Aimar, Modelling of filtration: From the polarised layer to deposit formation and compaction, Desalination, 145 (2002), 139-146.

[11] Y. Bessiere, P. Bacchin, Low fouling conditions in dead-end filtration : evidence for a critical filtered volume and interpretation using critical osmotic pressure, to be submitted to J. Membr. Sci.

[12] Y. Bessiere, P. Bacchin, B. Jefferson, Dead end filtration of natural organic matter: experimental evidence of critical conditions, Desalination, FCF 2004 